\title{
GRADIENT FLOWS ON KAČ-MOODY ALGEBRAS AND THE PERIODIC TODA LATTICE*
}

\author{
M.-A. BELABBAS ${ }^{\dagger}$
}

\begin{abstract}
In this paper, we provide a new formulation for the generalized periodic Toda lattice. Since the work of Kostant, Adler and Symes, it has been known that the Toda lattice is related to the structure of simple Lie algebras. Indeed, the non-periodic and the periodic Toda lattices can be expressed as Hamiltonian systems on coadjoint orbits: the former of a simple Lie group and the latter of the associated loop group. Alternatively, the non-periodic Toda lattice was expressed as a gradient flow on an adjoint orbit of a simple Lie group by Bloch, Brockett and Ratiu. Based on the description of certain gradient flows on adjoint orbits in affine Lie algebras as double bracket equations, we show that the periodic Toda lattice also admits a canonical gradient formulation and relate it to the structure of affine Kač-Moody algebras.
\end{abstract}

1. Introduction. The Toda lattice, which was introduced by M. Toda in 1967 to explain some then surprising computational experiments of Kruskal, has since had an impact that extends far beyond its original purpose. It appears, for example, in various areas of pure and applied mathematics, such as control theory [BC97], optimization [HM96], random matrix theory [Dei99], and others. One of the reasons behind this ubiquity is the existence of canonical formulations in terms of Lie algebraic data; these have provided key insights in the understanding of completely integrable systems, highlighted some of their symmetries and allowed for various generalizations [RS94].

In this paper, we establish a new canonical formulation for the periodic Toda lattice as a gradient flow on the adjoint orbit of a loop group on an affine Lie algebra. Thus similarly to its non-periodic counterpart, the periodic Toda lattice is a completely integrable gradient flow [BBR92]. Our second and equally important objective is to show that double-bracket flows are naturally generalized to affine algebras (See Theorem 2) and to use the Toda lattice as an example to demonstrate their relevance.

Within the body of work of control theory, we can see at least three different connections with the Toda lattice, and all are related, as we will briefly explain below, to the work of R. Brockett.

There was first the discovery by J. Moser [Mos] that the action-angle variables for the non-periodic Toda lattice are defined on the space $\operatorname{Rat}(n, n)$ of rational functions of McMillan degree $n$ and Cauchy index $n$. The basis for the study of this space had been laid independently by Brockett [Bro76] a few years earlier and the discovery

\footnotetext{
* Dedicated to Roger Brockett on the occasion of his 70th birthday.

†School of Engineering and Applied Sciences, Harvard University, Oxford St Cambridge MA 02138. E-mail: belabbas@fas.harvard.edu
} 
of Moser established new areas of application for this work. This in turn led to a further study of the relation between dynamics and rational functions [Kri79] and the topology of the space $\operatorname{Rat}(n, n)$ [Seg79]; see [Bro97] for a control theoretic point of view on the work of Moser.

Another connection is found in optimal control theory and the study of variational problems; a partial list of results in this area comprises the works of Brockett [Bro94, Bro99], Faybusovich [Fay88] and Bloch et al. [BC97]. A common thread among these works is the definition of classes of optimal control problems that admit explicit solutions as a direct consequence of their reduction to a system of Toda type.

A third connection with control theory also originated in work done by Brockett a few years before the link was made clear. We are thinking here of the work [Bro89, Bro91] on geometric pattern matching and double bracket flows which led to a new formulation of the non-periodic Toda lattice as a gradient flow on adjoint orbit by Bloch, Brockett and Ratiu [BBR90, BBR92]. This latter aspect of the Toda lattice will be the concern of this paper.

Consider the Hamiltonian

$$
H_{n p}=\frac{1}{2} \sum_{i=1}^{l} p_{i}^{2}+\sum_{i=1}^{l-1} e^{q_{i}-q_{i+1}}
$$

describing the motion of $l$ particles on the real line with unidirectional nearestneighbor interactions, where $q_{i}$ is the position of the $i^{t h}$ particle and $p_{i}$ its velocity. The link between the Toda lattice and the structure of simple Lie algebras was in some sense first suggested by Flaschka who, using the variables $\left(a_{i}=\right.$ $\left.1 / 2 e^{\left(q_{i}-q_{i+1}\right)}, b_{i}=-1 / 2 p_{i}\right)$ that now bear his name, expressed it in a Lax pair form [Fla74] $\dot{L}_{n p}=\left[L_{n p}, B_{n p}\left(L_{n p}\right)\right]$ with

$$
L_{n p}=\left[\begin{array}{cccc}
b_{1} & a_{1} & \ldots & 0 \\
a_{1} & b_{2} & \ddots & \vdots \\
\vdots & \ddots & \ddots & a_{l-1} \\
0 & \ldots & a_{l-1} & b_{l}
\end{array}\right] ; B_{n p}\left(L_{n p}\right)=\left[\begin{array}{cccc}
0 & a_{1} & \ldots & 0 \\
-a_{1} & 0 & \ddots & \vdots \\
\vdots & \ddots & \ddots & a_{l-1} \\
0 & \ldots & -a_{l-1} & 0
\end{array}\right]
$$

The relation was subsequently made precise in Kostant [Kos79], Adler [Adl79] and Symes [Sym80], where the non-periodic Toda lattice was explicitly linked to Dynkin diagrams [Sam90] and expressed as a Hamiltonian system on a coadjoint orbit.

The periodic Toda lattice corresponds to the Hamiltonian

$$
H_{p}=H_{n p}+e^{\left(q_{l}-q_{1}\right)}
$$

where the first and last particles are allowed to interact directly. The periodic Toda lattice was generalized to all simple Lie algebras by Bogoyavlensky [Bog76] and these generalized lattices were later expressed as Hamiltonian systems on coadjoint orbits 
of loop groups by Adler and van Moerbeke [AvM80]. This formulation results in the Lax pair with parameter:

(1) $L_{p}=\left[\begin{array}{cccc}b_{1} & a_{1} & \ldots & a_{l} z^{-1} \\ a_{1} & b_{2} & \ddots & \vdots \\ \vdots & \ddots & \ddots & a_{l-1} \\ a_{l} z & \ldots & a_{l-1} & b_{l}\end{array}\right] ; B_{p}\left(L_{p}\right)=\left[\begin{array}{cccc}0 & a_{1} & \ldots & -a_{l} z^{-1} \\ -a_{1} & 0 & \ddots & \vdots \\ \vdots & \ddots & \ddots & a_{l-1} \\ a_{l} z & \ldots & -a_{l-1} & 0\end{array}\right]$

The reason for introducing the parameter $z$, which may appear superfluous at first, will be made clear in Example 2 below.

While the Hamiltonian formulation is more common in pure mathematics and physics, a different point of view - which we will adopt here - was taken by Bloch et al. [BBR90] to show that, quite surprisingly, the generalized non-periodic Toda lattices admit a canonical gradient formulation as a so-called double-bracket flow. As mentioned above, these flows were introduced independently [Bro91] and have since then found many applications in engineering, signal processing, computer vision and applied mathematics, see e.g. [BB06, Bro93] for references.

The proofs of the main results of this paper require mostly well-known facts about loop groups and affine algebras; these can be found for example in [Kac94, PS88]. One notable exception is in Section 3, where the use of the group of loops of Sobolev class $H^{1}$, which is an honest Hilbert manifold, is needed in order to define the normal metric and the associated gradient flows. The relevant background can be found in the works of Atiyah and Pressley [AP83] and Segal [Seg81],

It is also worth noting that, when compared with the Hamiltonian formulation, the description of the periodic Toda lattice exhibited here requires an additional variable $b_{0}$ corresponding to the imaginary root of the simple root system of the underlying affine Lie algebra. This will in turn correspond to an additional flow equation on the central extension of the algebra. To be more specific, let $\tilde{\mathfrak{g}}$ be the affine Kač-Moody algebra associated with a complex simple finite dimensional Lie algebra ${ }^{1} \mathfrak{g}$ of rank $l$. Let $C_{\text {aff }}$ be the Cartan matrix of $\tilde{\mathfrak{g}},\langle.,$.$\rangle be an invariant non-degenerate bilinear form,$ $E_{i j}$ be the matrix with entries zero everywhere except for the $(i, j)$ entry which is one, and $\mathbf{1}$ be the vector whose entries are all one. An element of $\tilde{\mathfrak{g}}$ is represented by a triplet $L \triangleq L_{0} \oplus u \mathrm{c} \oplus v$ d where $L_{0} \in \mathbb{C}\left[z, z^{-1}\right] \otimes \mathfrak{g}, u, v \in \mathbb{C}$, c is a central extension to $\mathbb{C}\left[z, z^{-1}\right] \otimes \mathfrak{g}$ and $\mathrm{d}$ is a derivation acting on $\mathbb{C}\left[z, z^{-1}\right] \otimes \mathfrak{g}$. For $\left\{H_{i}\right\}$ a set of simple coroots of $\tilde{\mathfrak{g}}$ and $e_{i}$ in the associated root spaces, we have

TheOREm 1. Let $x=\left(x_{0}, \ldots, x_{l}\right) \in \mathbb{R}^{l+1}$ be the unique solution of

$$
\left(C_{\text {aff }}+E_{11}\right) x=-\mathbf{1} .
$$

\footnotetext{
${ }^{1}$ see the next section for details on the notation and recalls of the background material.
} 
Set $N=\sum_{i=0}^{l} x_{i} H_{i}+x_{0}\left(z \frac{d}{d z}\right)$ and $L=\sum_{i=0}^{l} b_{i} H_{i}+\sum_{i=0}^{l} a_{i}\left(e_{i}+e_{-i}\right)$ with $a_{i}, b_{i} \in \mathbb{R}$.

Then $x_{0}=-c_{\mathfrak{g}}$, the Coxeter number of $\mathfrak{g}$, and the periodic Toda lattice associated with $\mathfrak{g}$ is the gradient flow for the function $\langle L, N\rangle$ with respect to the normal metric. Moreover, the flow can be written in the double-bracket form

$$
\dot{L}=[L,[L, N]] .
$$

In the first part of this paper, we recall some well-known facts about finitedimensional Lie algebras and establish the notation used. We then describe the structure of affine Kač-Moody algebras in some detail since they are not as common as their finite-dimensional counterparts in most areas of applied mathematics. In the following section we introduce the normal metric on an adjoint orbit of a loop group on an affine algebra and prove Theorem 1. In the last section, we write down explicitly the flows for the 4 infinite families of affine Lie algebras $\tilde{A}_{n}, \tilde{B}_{n}, \tilde{C}_{n}, \tilde{D}_{n}$.

\section{Loop groups and Kač-Moody Lie Algebras.}

2.1. Some notation and facts about finite-dimensional Lie algebras. We introduce in this section some notation and recall a few facts about finite-dimensional simple Lie algebras [Sam90] that will be of use in this paper.

Let $\mathfrak{g}$ be a finite dimensional semi-simple Lie algebra and $\mathfrak{h}$ a Cartan subalgebra of $\mathfrak{g}$. For $A, B \in \mathfrak{g}$, we denote by $[A, B]$ the Lie bracket of $A$ and $B$. We also write $\operatorname{ad}_{A}$ for $[A, \cdot]$. Since $\mathfrak{g}$ is simple, it admits a canonical non-degenerate symmetric invariant bilinear form, the Killing form, denoted by

$$
B(., .): \mathfrak{g} \times \mathfrak{g} \rightarrow \mathbb{C} .
$$

This form is moreover non-degenerate when restricted to a Cartan subalgebra.

Let $\alpha \in \mathfrak{h}^{*}$ and define

$$
\mathfrak{g}_{\alpha}=\left\{A \in \mathfrak{g} \text { s.t. } \operatorname{ad}_{H} A=\alpha(H) A \text { for all } H \in \mathfrak{h}\right\} .
$$

If $\mathfrak{g}_{\alpha} \neq \emptyset$ then $\alpha$ is a root and $\mathfrak{g}_{\alpha}$ is the associated root space. We will denote by $\Delta \subset \mathfrak{h}^{*}$ the set of roots, by $\Delta^{+} \subset \Delta$ a set of positive roots and by $\Delta^{0}$ a set of positive simple roots. The cardinality of $\Delta^{0}$ is called the rank of $\mathfrak{g}$. The root spaces are one-dimensional and together with $\mathfrak{h}$, they yield the root space decomposition of $\mathfrak{g}:$

$$
\mathfrak{g}=\mathfrak{h} \oplus \bigoplus_{\alpha \in \Delta} \mathfrak{g}_{\alpha}
$$

where $\oplus$ indicates a direct sum.

Let $\alpha \in \Delta$ and $\Delta^{0}=\left\{\alpha_{1}, \ldots \alpha_{l}\right\}$. A central fact about the structure of semisimple Lie algebra is that we can write $\alpha$ uniquely as

$$
\alpha=\sum_{i} m_{\alpha, i} \alpha_{i}
$$


with $m_{\alpha, i} \in \mathbb{Z}^{+}$if $\alpha \in \Delta^{+}$and $m_{\alpha, i} \in \mathbb{Z}^{-}$otherwise. The integer $|\alpha|=\sum_{i}\left|m_{\alpha, i}\right|$ is the height of $\alpha$. The root of maximal height in $\Delta^{+}$is unique and plays a distinguished role, we denote it by $\gamma$.

Let $e_{\alpha} \in \mathfrak{g}_{\alpha}, e_{-\alpha} \in \mathfrak{g}_{-\alpha}$ be such that $B\left(e_{\alpha}, e_{-\alpha}\right)=1$. Let $h_{\alpha}=\left[e_{\alpha}, e_{-\alpha}\right]$, the coroot associated to $\alpha$ is

$$
H_{\alpha}=\frac{2}{B\left(h_{\alpha}, h_{\alpha}\right)} h_{\alpha} \in \mathfrak{h}
$$

Another object from the structure theory of finite dimensional Lie algebras that we need is the Cartan matrix. Let $H_{\alpha_{i}}$ be the coroot of $\alpha_{i}$ (we sometimes use $H_{i}$ instead of $H_{\alpha_{i}}$ ). Recall that the simple roots span $\mathfrak{h}^{*}$ and since the Killing form is nondegenerate on $\mathfrak{h}$, it defines an inner product on $\mathfrak{h}^{*}$, denoted by $B^{*}$. The Cartan matrix $C$ of $\mathfrak{g}$ is then given by

$$
C_{i j} \triangleq \frac{2 \mathrm{~B}^{*}\left(\alpha_{i}, \alpha_{j}\right)}{\mathrm{B}^{*}\left(\alpha_{i}, \alpha_{i}\right)}=\alpha_{i}\left(H_{\alpha_{j}}\right)
$$

ExAmple $1(\mathfrak{s l}(l))$. Let $\mathfrak{g}=\mathfrak{s l}(l)$ and $E_{i j}$ be the matrix with a one at the $(i, j)$ position and zeroes everywhere else. The space $\mathfrak{h}$ of traceless $(l \times l)$ diagonal matrices is a Cartan subalgebra of $\mathfrak{g}$. Let $d_{i}$ be the element of $\mathfrak{h}^{*}$ which extracts the $i^{\text {th }}$ diagonal entry, i.e. $d_{i}\left(E_{j j}\right)=\delta_{i j}$. With this notation, a set of roots is given by $\Delta=\left\{ \pm\left(d_{i}-d_{j}\right)\right\}$. The coroots are given by $\pm\left(E_{i i}-E_{j j}\right)$. A set of simple roots will be $\Delta_{0}=\left\{\alpha_{1}=d_{1}-d_{2}, \alpha_{2}=d_{2}-d_{3}, \ldots, \alpha_{l-1}=d_{l-1}-d_{l}\right\}$ and the associated root spaces are given by $\mathfrak{g}_{d_{i}-d_{j}}=E_{i j}$. The highest root is $\alpha_{1}+\alpha_{2}+\ldots+\alpha_{l-1}=d_{1}-d_{l}$. The corresponding coroot is $E_{11}-E_{l, l}$ which admits $E_{1, l}$ as root space.

2.2. Loop groups and loop algebras. An infinite dimensional Lie group is a group for which the multiplication and inverse operations are smooth and which is at the same time an infinite-dimensional manifold. While in case the group is a Banach manifold there exists a well-developed theory [Bou72], which is rather similar to the theory of finite dimensional Lie groups, this is not the case in more general settings. We consider here another class of groups for which a well-developed theory exists, the groups of smooth maps from the circle $S^{1}$ to a Lie group.

Let $G$ be a finite-dimensional simple Lie group and $L G$, called the loop group of $G$, be the space of smooth maps from $S^{1}$ to $G$ with the group operation being point-wise multiplication:

$$
L G=\left\{\Phi: S^{1} \rightarrow G \mid \Phi \text { is smooth }\right\}
$$

and if $\Phi_{1}(\theta), \Phi_{2}(\theta), \Phi_{3}(\theta) \in L G$ with $\Phi_{3}=\Phi_{1} \Phi_{2}$ then $\Phi_{3}(\theta)=\Phi_{1}(\theta) \Phi_{2}(\theta)$.

As a topological space, $L G$ is an infinite dimensional manifold for the topology of uniform convergence for $\Phi$ and all its derivatives [PS88]. The identity element of $L G$ is the constant loop $\phi_{I}: S^{1} \rightarrow I$ where $I$ is the identity in $G$. By considering a 
small perturbation to $\phi_{I}$, one can easily be convinced that the Lie algebra of $L G$ is

$$
L \mathfrak{g}=\left\{\phi: S^{1} \rightarrow \mathfrak{g} \mid \phi \text { is smooth }\right\}
$$

and that the exponential map

$$
\exp : L \mathfrak{g} \rightarrow L G
$$

is well-defined.

Any element $A(z) \in L \mathfrak{g}$ can be written in terms of its Fourier series as

$$
A(z)=\sum_{i \in \mathbb{Z}} A_{i} z^{i}
$$

with $A_{i} \in \mathfrak{g}$. The Lie bracket of two elements $A, B \in L \mathfrak{g}$ is then

$$
[A, B]=\sum_{i, j}\left[A_{i}, B_{j}\right] z^{i+j} .
$$

It is sometimes helpful to think of a loop algebra in the following way: to each element $A(z)$ we associate the infinite dimensional matrix

$$
m(A)=\left[\begin{array}{lllllll}
\ddots & \vdots & & & & \\
\ldots & A_{0} & A_{1} & \ldots & & \\
\ldots & A_{-1} & A_{0} & A_{1} & A_{2} & \ldots \\
\ldots & A_{-2} & A_{-1} & A_{0} & A_{1} & \ldots \\
& & & A_{-1} & A_{0} & \ldots \\
& & & & \vdots & \ddots
\end{array}\right]
$$

where an arbitrary basepoint is chosen. We see that $m([A, B])=[m(A), m(B)]=$ $m(A) m(B)-m(B) m(A)$ and hence $m$ is a Lie algebra isomorphism.

Example 2 (The periodic Toda lattice). Recall the $\tilde{A}_{n}$ periodic Toda lattice whose Lax pair was given in Equation (1), it is easy to see that

$$
m(L)=\left[\begin{array}{cccccccc}
\ddots & \ddots & & & & & & \\
\ddots & b_{1} & a_{1} & & & & & \\
& a_{1} & b_{2} & \ddots & & & & \\
& & \ddots & \ddots & a_{l-1} & & & \\
& & & a_{l-1} & b_{l} & a_{l} & & \\
& & & & a_{l} & b_{1} & a_{1} & \\
& & & & & a_{1} & b_{2} & \ddots \\
& & & & & & \ddots & \ddots
\end{array}\right] .
$$


Thus considering the periodic Toda lattice as a flow on a loop algebra is equivalent to "unfolding" the matrix into an infinite dimensional periodic tridiagonal matrix.

Any symmetric bi-invariant non-degenerate form B on $\mathfrak{g}$ defines one on $L \mathfrak{g}$ according to

$$
\left\langle(A(z), B(z)\rangle=\frac{1}{2 \pi i} \oint \mathrm{B}(A(\theta), B(\theta)) d \theta=\sum_{j+k=0} \mathrm{~B}\left(A_{j}, B_{k}\right)\right.
$$

where $z=e^{i \theta}$.

We now make a key observation: let $d$ be the height of the highest root of $\mathfrak{g}$. This number is related to the Coxeter number (i.e. the number of roots of $\mathfrak{g}$ divided by its rank) $c_{\mathfrak{g}}$ of $\mathfrak{g}: d+1=c_{\mathfrak{g}}$. We will show that we can obtain for a loop algebra a grading which is analogous to the one of finite dimensional simple Lie algebras. Define

$$
L \mathfrak{g}_{i} \triangleq \bigoplus_{\substack{k+j d=i \\|k| \leq d-1}} z^{j} \mathfrak{g}_{k}
$$

We have $L \mathfrak{g}=\bigoplus_{i} L \mathfrak{g}_{i}$ and

$$
\begin{aligned}
{\left[L \mathfrak{g}_{i}, L \mathfrak{g}_{1}\right] } & =\left[\bigoplus_{\substack{k+j d=i \\
|k| \leq d-1}} z^{j} \mathfrak{g}_{k}, \mathfrak{g}_{1}+z \mathfrak{g}_{-(d-1)}\right] \\
& =\bigoplus_{\substack{k+j d=i \\
|k| \leq d-1}} z^{j} \mathfrak{g}_{k+1} \oplus \bigoplus_{\substack{k+j d=i \\
|k| \leq d-1}} z^{j+1} \mathfrak{g}_{k-d+1} \\
& =\bigoplus_{\substack{k+j d=i+1 \\
|k| \leq d-1}} z^{j} \mathfrak{g}_{k}=L \mathfrak{g}_{i+1} .
\end{aligned}
$$

From this observation we deduce that a Cartan subalgebra of $L \mathfrak{g}$ is $L \mathfrak{g}_{0}$ and the corresponding simple root space is $L \mathfrak{g}_{1}=\mathfrak{g}_{1}+z \mathfrak{g}_{-(d-1)}$

2.3. Affine Kač-Moody Lie Algebras. Loop groups and loop algebras behave in a remarkably similar manner to their finite dimensional counterparts. An important difference, which is essential to our description of the Toda lattice, is the existence of canonical non-trivial central extensions.

Let $\mathbb{C}\left[z^{-1}, z\right]$ denote the Laurent polynomials in the complex variable $z$. We have $L \mathfrak{g}=\mathbb{C}\left[z^{-1}, z\right] \otimes_{\mathbb{C}} \mathfrak{g}$. The Kač-Moody Lie algebra $\tilde{\mathfrak{g}}$ associated to $\mathfrak{g}$ is obtained by adding two copies of $\mathbb{C}$ to $L \mathfrak{g}$ in a way we describe now:

A. A cocycle is a skew-symmetric bilinear form on the Lie algebra which also respects the Jacobi identity. More precisely, $\omega: \mathfrak{g} \times \mathfrak{g} \rightarrow \mathbb{C}$ is a cocycle if for $A, B, C \in \mathfrak{g}$ the following is true:

$$
\begin{array}{r}
\omega(A, B)+\omega(B, A)=0 \\
\omega([A, B], C)+\omega([B, C], A)+\omega([C, A], B)=0
\end{array}
$$


Given a cocycle, we can obtain a central extension of $\mathfrak{g}$ by adding a copy of $\mathbb{C}$ and using the following bracket:

$$
\left[\left(A \oplus x_{1}\right),\left(B \oplus y_{1}\right)\right]=[A, B] \oplus \omega(A, B) \mathrm{c}
$$

where $x_{1}, y_{1} \in \mathbb{C}$ and with a slight abuse of notation, we also denoted the new bracket by [.,.]. It can be shown [PS88] that for $\mathfrak{g}$ semisimple, essentially all the cocycles in $L \mathfrak{g}$ are of the form

$$
\omega(A, B)=\frac{1}{2 \pi i} \oint \mathrm{B}\left(A^{\prime}, B\right) d \theta
$$

B. Let $a \in \mathbb{C}$, consider the differential operator $a z \frac{d}{d z}$ acting on the centrally extended loop algebra $\mathbb{C}\left[z^{-1}, z\right] \otimes \mathfrak{g} \oplus \mathbb{C} c$

$$
\operatorname{ad}_{a z \frac{d}{d z}}(A(z) \oplus u \mathrm{c})=a z \frac{d A}{d z} \oplus 0 .
$$

This operator sends the center and constant elements of $L \mathfrak{g} \oplus \mathbb{C}$ to zero. It is easy to see that its eigenspaces are of the form $A z^{i}$ where $A$ is a constant. We use the notation $\mathrm{d} \triangleq z \frac{d}{d z}$.

We are now ready to define the Kač-Moody Lie algebra associated with $\mathfrak{g}$. It is the complex vector space

$$
\tilde{\mathfrak{g}} \triangleq\left(\mathbb{C}\left[z^{-1}, z\right] \otimes_{\mathbb{C}} \mathfrak{g}\right) \oplus \mathbb{C} \mathrm{c} \oplus \mathbb{C d}
$$

with the Lie bracket

(6) $\left[A_{1} \oplus u_{1} \mathrm{c} \oplus v_{1} \mathrm{~d}, A_{2} \oplus u_{2} \mathrm{c} \oplus v_{2} \mathrm{~d}\right]=\left(\left[A_{1}, A_{2}\right]+v_{1} z \frac{d A_{2}}{d z}-v_{2} z \frac{d A_{1}}{d z}\right) \oplus \omega(A, B) \mathrm{c} \oplus 0 \mathrm{~d}$

We equip $\tilde{\mathfrak{g}}$ with a non-degenerate bilinear form $\langle.,\rangle:. \tilde{\mathfrak{g}} \times \tilde{\mathfrak{g}} \rightarrow \mathbb{C}$ defined as follows:

$$
\left\langle A_{1} \oplus u_{1} \mathrm{c} \oplus v_{1} \mathrm{~d}, A_{2} \oplus u_{2} \mathrm{c} \oplus v_{2} \mathrm{~d}\right\rangle=\frac{1}{2 \pi i} \oint \mathrm{B}\left(A_{1}, A_{2}\right) d \theta+u_{1} v_{2}+u_{2} v_{1}
$$

where the integration is on the unit circle in $\mathbb{C}$. It can be shown that this form is ad-invariant for the bracket of Equation (6).

We now establish some facts about the structure of $\tilde{\mathfrak{g}}$ and how it relates to the structure of $\mathfrak{g}$. If $\mathfrak{h}$ is a Cartan subalgebra of $\mathfrak{g}$ then it is also a commutative diagonalizable subalgebra of $\tilde{\mathfrak{g}}$ and, in view of the bracket of Equation (6), elements in $\mathbb{C} c$ or in $\mathbb{C} d$ commute with elements of $\mathfrak{h}$. Since $\mathbb{C} c$ is the center, it is clearly diagonalizable. Summarizing this we have that [Kac94]

$$
\tilde{\mathfrak{h}}=\mathfrak{h} \oplus \mathbb{C c} \oplus \mathbb{C d}
$$


is a maximal commutative diagonalizable subalgebra of $\tilde{\mathfrak{g}}$. Thinking of $\mathfrak{h}^{*}$ as included in $\tilde{\mathfrak{h}}^{*}$, we take the convention that for $\alpha \in \mathfrak{h}^{*}, \alpha(\mathrm{c})=\alpha(\mathrm{d})=0$. We write the duals of $\mathrm{d}$ and c as $\delta$ and $\mathrm{c}^{*}$ respectively. For $H \in \mathfrak{h}, A \in \mathfrak{g}_{\alpha}$ we have

$$
\operatorname{ad}_{H} z^{j} A=\alpha(H) z^{j} A
$$

and, as mentioned before,

$$
\operatorname{ad}_{z \frac{d}{d z}} A z^{j}=j z^{j} A
$$

Hence the root space decomposition of $\tilde{\mathfrak{g}}$ is given by

$$
\tilde{\mathfrak{g}}=\tilde{\mathfrak{h}} \oplus \sum_{j \in \mathbb{Z}, \alpha \in \Delta} z^{j} \mathfrak{g}_{\alpha}
$$

We thus have a copy of the root spaces of the underlying finite dimensional algebra for each power of $z$. We point out that the root spaces are finite dimensional, and this is due to the introduction of the derivation $\mathrm{d}$.

The set of roots of the Kač-Moody algebra $\tilde{\mathfrak{g}}$ is given by

$$
\tilde{\Delta}=\{j \delta+\alpha, j \in \mathbb{Z}, \alpha \in \Delta \cup 0\}-\{0\}
$$

The roots $j \delta+\alpha$ are called real roots and the dimension of the associated root spaces is one, since it is a copy of the root space associated with $\alpha \in \Delta$. The roots $j \delta$ are called imaginary roots and the dimension of the root space is $l$, the rank of $\mathfrak{g}$. A set of simple roots $\tilde{\Delta}_{0}$ is given by

$$
\tilde{\Delta}_{0}=\left\{\delta-\gamma, \alpha_{1}, \ldots, \alpha_{r}\right\}
$$

where $\gamma$ is the highest root of $\mathfrak{g}$ and $\alpha_{1}, \ldots, \alpha_{r}$ are simple roots of $\mathfrak{g}$. The general convention is to write $\alpha_{0}=\delta-\gamma$.

The inner product $\langle.,$.$\rangle of Equation (7) is non-degenerate when restricted to \tilde{\mathfrak{h}}$ and $\left\langle\tilde{\mathfrak{g}}_{\alpha}, \tilde{\mathfrak{g}}_{\beta}\right\rangle=0$ if $\alpha+\beta \neq 0$ and thus we can define the coroots and Cartan matrix of $\tilde{\mathfrak{g}}$ similarly to what is done in the finite dimensional case. The Cartan matrix of an affine Kač-Moody algebra can be degenerate but satisfies other properties of Cartan matrices. It moreover has as a submatrix the Cartan matrix of $\mathfrak{g}$ and we take the convention of naming the additional row/column $C_{0, .}$ and $C_{., 0}$.

EXAMPLE $3(\tilde{\mathfrak{s}}(l))$. We take $\mathfrak{g}=\mathfrak{s l}(l)$, a set of simple roots and the corresponding highest root were given in Example 1. Hence, the set of simple roots of the associated Kač-Moody algebra is

$$
\tilde{\Delta}_{0}=\left\{\delta-\left(d_{1}-d_{l}\right), d_{1}-d_{2}, \ldots, d_{l-1}-d_{l}\right\}
$$


The Cartan matrix of $\mathfrak{s l}(l)$ is given by the following $(l-1) \times(l-1)$ matrix:

$$
C=\left(\begin{array}{ccccc}
2 & -1 & 0 & \ldots & 0 \\
-1 & 2 & -1 & & \vdots \\
0 & -1 & \ddots & \ddots & 0 \\
\vdots & & \ddots & & -1 \\
0 & \ldots & 0 & -1 & 2
\end{array}\right)
$$

By abuse of notation, we will denote by $\langle.,$.$\rangle the inner product defined in Equa-$ tion (7) as well as the inner product it naturally induces on $\tilde{\mathfrak{g}}^{*}$.

We need to evaluate $C_{\mathrm{aff}, 0, i}$ and $C_{\mathrm{aff}, i, 0}$ for $i=0 \ldots l-1$. First, we observe that $\left\langle\delta, \alpha_{i}\right\rangle=0$. We have $C_{\mathrm{aff}, 0,0}=2 \frac{\left\langle\alpha_{0}, \alpha_{0}\right\rangle}{\left\langle\alpha_{0}, \alpha_{0}\right\rangle}=2$ since $\left\langle\alpha_{0}, \alpha_{0}\right\rangle=\sum_{i}\left\langle\alpha_{i}, \alpha_{i}\right\rangle>0$. Next,

$$
\begin{aligned}
\left\langle\alpha_{0}, \alpha_{1}\right\rangle & =\left\langle\delta, \alpha_{1}\right\rangle-\left\langle\alpha_{1}, \alpha_{1}\right\rangle-\left\langle\alpha_{1}, \alpha_{2}\right\rangle-\ldots-\left\langle\alpha_{1}, \alpha_{r}\right\rangle \\
& =0-2-(-1) \\
& =-1
\end{aligned}
$$

where we used the fact that $\left\langle\alpha_{i}, \alpha_{i}\right\rangle=2,\left\langle\alpha_{i}, \alpha_{j}\right\rangle=-1$ if $|i-j|=1$.

For $i=2 \ldots n-2\left\langle\alpha_{0}, \alpha_{i}\right\rangle=-2+1+1=0$ and $\left\langle\alpha_{0}, \alpha_{l-1}\right\rangle=-1$. Gathering these facts, we have

$$
C_{\mathrm{aff}}=\left(\begin{array}{ccccc}
2 & -1 & 0 & \ldots & -1 \\
-1 & 2 & -1 & & \vdots \\
0 & -1 & \ddots & \ddots & 0 \\
\vdots & & & & -1 \\
-1 & \ldots & 0 & -1 & 2
\end{array}\right)
$$

3. Adjoint orbits, the normal metric and Toda flows. The focus of this section is on the description of gradient flows on adjoint orbits of loop groups through elements of an affine Lie algebra.

3.1. Adjoint orbits and compact form. We start by defining the adjoint action of $L G$ on $\tilde{\mathfrak{g}}$. In order to avoid an unnecessary proliferation of symbols, we use the notation $\operatorname{Ad}_{g}$ to denote the action of $L G$ on both $L \mathfrak{g}$ and $\tilde{\mathfrak{g}}$.

Proposition 1. [PS88] The adjoint action of LG on $\tilde{\mathfrak{g}}$ is given by

$$
\begin{aligned}
& \operatorname{Ad}_{g}(A \oplus u \mathrm{c} \oplus v \mathrm{~d})=\operatorname{Ad}_{g} A-v z \frac{d g}{d z} g^{-1} \\
& \oplus\left(u+\left\langle g^{-1} z \frac{d g}{d z}, A\right\rangle-\frac{1}{2} v\left\langle g^{-1} z \frac{d g}{d z}, g^{-1} z \frac{d g}{d z}\right\rangle\right) \mathrm{c} \oplus v \mathrm{~d}
\end{aligned}
$$


The adjoint orbit of $L G$ at $L \in \tilde{\mathfrak{g}}$ is

$$
\mathcal{O}_{L} \triangleq\left\{A \in \tilde{\mathfrak{g}} \mid A=\operatorname{Ad}_{g} L \text { for } g \in L G\right\} .
$$

We observe that the term $v \mathrm{~d}$ is left constant and that the adjoint action on elements of the form $A_{0} \oplus u \mathrm{c} \oplus 0 \mathrm{~d}$ reduces to

$$
\operatorname{Ad}_{g}\left(A_{0} \oplus u \mathrm{c}\right)=\operatorname{Ad}_{g} A_{0} \oplus\left(u+\left\langle g^{-1} z \frac{d g}{d z}, A_{0}\right\rangle\right) \mathrm{c} .
$$

The tangent space to the orbit at a point $L$, termed $T \mathcal{O}_{L}$, is spanned by vectors of the form $\operatorname{ad}_{L} X$ for $X \in L \mathfrak{g}$ :

$$
T \mathcal{O}_{L}=\{[L, X] \mid X \in L \mathfrak{g}\} .
$$

Let $\tilde{\mathfrak{g}}^{\mathbb{R}}$ (resp. $L \mathfrak{g}^{\mathbb{R}}$ ) be the realification of $\tilde{\mathfrak{g}}$ (resp. $L \mathfrak{g}$.) From Equation (9), we know that we can write $A \in L \mathfrak{g}$ as

$$
A=\sum_{i=1}^{l} b_{i} H_{i}+\sum_{j \in \mathbb{Z}, \alpha \in \Delta} a_{j, \alpha} e_{\alpha} z^{j} .
$$

We define the symmetrics of $L \mathfrak{g}^{\mathbb{R}}$ as

$$
\mathfrak{k}=\sum_{i=1}^{l} b_{i} H_{i}+\sum_{j \geq 0, \alpha \in \Delta^{+}} a_{j, \alpha}\left(e_{\alpha} z^{j}+e_{-\alpha} z^{-j}\right)
$$

and the skew-symmetrics as

$$
\mathfrak{k}^{\perp}=\sum_{i \geq 0, \alpha \in \Delta^{+}} a_{j, \alpha}\left(e_{\alpha} z^{j}-e_{-\alpha} z^{-j}\right),
$$

where all the coefficients are real. Observe that

$$
\begin{array}{r}
\left\langle\sum_{i=1}^{l} b_{i} H_{i}+\sum_{j \geq 0, \alpha \in \Delta^{+}} a_{j, \alpha}\left(e_{\alpha} z^{j}+e_{-\alpha} z^{-j}\right), \sum_{j \geq 0, \alpha \in \Delta^{+}} a_{j, \alpha}^{\prime}\left(e_{\alpha} z^{j}-e_{-\alpha} z^{-j}\right)\right\rangle \\
=\sum_{j, \alpha} a_{j, \alpha} a_{j, \alpha}^{\prime}-a_{j, \alpha}^{\prime} a_{j, \alpha}=0
\end{array}
$$

and thus these two subspaces are orthogonal:

$$
\left\langle\mathfrak{k}, \mathfrak{k}^{\perp}\right\rangle=0 .
$$

The definition of the compact real form of $L \mathfrak{g}$ is similar to the one in the finitedimensional case [Sam90]:

$$
\mathfrak{k}_{u} \triangleq i \mathbb{R} \mathfrak{k}+\mathbb{R k}^{\perp} .
$$

We can easily see using Equation (11) that $\mathfrak{k}_{u}$ is a real form of $L \mathfrak{g}$; by the following Proposition, it is indeed compact:

Proposition 2. The bilinear form $\langle.,$.$\rangle is negative definite on \mathfrak{k}_{u}$.

The proof of Proposition 2 follows the lines of the finite dimensional case [Sam90], so we omit it here. 
3.2. The normal metric. In order to generalize the normal metric [Ati82, Bro93] to the infinite-dimensional setting we are concerned with, we need a larger group than the group of smooth maps from $S^{1}$ to $G$. Namely, we consider the group $L G^{1}$ of maps $\Phi: S^{1} \rightarrow G$ which are of Sobolev class $H^{1}$ (that is for any representation of $G$ as a matrix group, the entries of $\Phi$ are in $H^{1}$.) Similarly, we let $L \mathfrak{g}^{1}$ be the Hilbert space $H^{1}\left(S^{1}, \mathfrak{g}\right)$. It can be shown that $L G$ is dense in $L G^{1}$ [AP83] and that the exponential map exp : $L \mathfrak{g}^{1} \rightarrow L G^{1}$ is a local diffeomorphism onto a neighborhood of the identity.

Let $L K_{u}^{1}$ be the loop group associated to the loop algebra $\mathfrak{k}_{u}^{1}$ and let $\mathcal{O}_{L}^{K_{u}^{1}}$ be the adjoint orbit obtained by the action of $L K_{u}^{1}$ on $L=L_{0} \oplus u c \in \mathfrak{k}_{u}^{1} \oplus \mathbb{R}$. We denote by $\mathfrak{k}_{u}^{0}$ the algebra of loops of class $H^{0}$ (or equivalently $L^{2}$, i.e. bounded for the metric of Equation (7)). We have that $\mathfrak{k}_{u}^{1} \subset \mathfrak{k}_{u}^{0}$ with a dense and continuous embedding. The definition of the normal metric will rely on the inner product of $\mathfrak{k}_{u}^{0}$, which is given in Equation (3). The reason behind this choice is that there are no bi-invariant metric on $\mathfrak{k}_{u}^{1}[\mathrm{AP} 83]$.

To make the construction of the normal metric more transparent, we take the convention in this section that $\left[L_{0}, \cdot\right]$ maps $\mathfrak{k}_{u}^{0}$ to itself (we thus omit here the central extension) and $\operatorname{ad}_{L_{0}} W=\left[L_{0}, W\right] \oplus \omega\left(L_{0}, W\right)$ c. Since $L_{0}$ is of class $H^{1},\left[L_{0}, \cdot\right]$ and $\omega\left(L_{0}, \cdot\right)$ are bounded; as a consequence, $\operatorname{ker}\left[L_{0}, \cdot\right]$ is closed and, by the Riesz representation theorem, there is a unique $\omega_{L}$ such that $\omega\left(L_{0}, \cdot\right)=-\left\langle\omega_{L}, \cdot\right\rangle$. It is obviously given by

$$
\omega_{L}=-z \frac{d L_{0}}{d z}
$$

We restrict the domain of $\left[L_{0}, \cdot\right]$ to ker $\left[L_{0}, \cdot\right]^{\perp}$ and write its inverse (which is well-defined there) as $\left[L_{0}, \cdot\right]^{-1}$.

The case of $\operatorname{ad}_{L}$ is only slightly more involved: let $\pi \omega_{L}$ be the orthogonal projection of $\omega_{L}$ onto ker $\left[L_{0}, \cdot\right]$ with respect to the $H^{0}$ metric. We have that $\omega\left(L_{0}, \cdot\right)$ is invertible when restricted to $\pi \omega$; we denote its inverse by $\omega^{-1}\left(L_{0}, \cdot\right)$. We restrict $\operatorname{ad}_{L}$ to its coimage, which is isomorphic to ker $\left[L_{0}, \cdot\right]^{\perp} \oplus \pi \omega_{L}$ and

$$
\operatorname{ad}_{L}^{-1}\left(W_{0} \oplus w \mathrm{c}\right)=\left[L_{0}, W_{0}\right]^{-1}+\omega^{-1}\left(L_{0}, w_{0}-\omega\left(L,\left[L_{0}, W_{0}\right]^{-1}\right)\right),
$$

and it is easy to see that $\operatorname{ad}_{L}^{-1} \operatorname{ad}_{L}=\mathrm{I}$.

Owing to the above, we can formulate

Definition 1 (Normal metric). Let $Y, Z \in T \mathcal{O}_{L}^{K_{u}^{1}}$. The normal metric is defined $a s$

$$
\langle Y, Z\rangle_{N} \triangleq\left\langle\operatorname{ad}_{L}^{-1} Y, \operatorname{ad}_{L}^{-1} Z\right\rangle
$$

Proposition 3. Let $L=L_{0} \oplus u c \in \mathfrak{k}_{u}^{1} \oplus \mathbb{R} c$. The normal metric is non-degenerate on $\mathcal{O}_{L}^{K_{u}^{1}}$ 
Proof. This follows from Proposition 2 and the above discussion.

REMARK 1. A sufficient condition for $\operatorname{ad}_{L}^{-1}$ to be bounded is that the spectrum of $L_{0}$ be simple for all $z \in S^{1}$. To see this, first we notice that $L_{0}$ can be represented as a Hermitian matrix and is diagonalizable by a unitary matrix $U(z)$ for all $z$ : $L_{0}(z)=U D U^{\dagger}, D$ diagonal. Since the unitary group is simply connected, there exists a map [BT95] $U: S^{1} \rightarrow L U$ which diagonalizes $L_{0}$. Let

$$
\|X\|=\oint B(X, X) d \theta
$$

By invariance of the inner product, we have for $X \in \operatorname{ker}\left[L_{0}, \cdot\right]^{\perp}$,

$$
\begin{aligned}
\left\|\left[L_{0}, X\right]\right\| & =\left\|\left[U L_{0} U^{\dagger}, U X U^{\dagger}\right]\right\| \\
& =\left\|\left(\tilde{X}_{i j}\left(D_{i i}-D_{j j}\right)\right)_{i j}\right\|
\end{aligned}
$$

where $\tilde{X}=U X U^{\dagger}$. If the spectrum of $L_{0}$ is simple for all $z$, then defining $c \triangleq$ $\min _{i, j} \min _{z \in S^{1}}\left|D_{i i}(z)-D_{j j}(z)\right|^{2}>0$ we have the inequality

$$
\left\|\left[L_{0}, X\right]\right\| \geq c\|X\| .
$$

The constant $c$ depends only on the adjoint orbit.

We proceed by showing that gradient flows on adjoint orbits with respect to the normal metric can be written in a double bracket form.

Let $L=L_{0} \oplus u$ c and $V: \tilde{\mathfrak{g}}^{1, \mathbb{R}} \rightarrow \mathbb{R}$ a smooth real function. We denote by $\nabla^{N} V$ the gradient of $V$ with respect to the normal metric, i.e. $\nabla^{N} V$ satisfies

$$
d V \cdot X=\left\langle\nabla^{N} V(L), X\right\rangle_{N} \text { for all } X \in T \mathcal{O}_{L}^{K_{u}^{1}}
$$

and by $\nabla V$ the gradient of $V$ with respect to $\langle.,$.$\rangle .$

THEOREM 2. The gradient of $V$ on an adjoint orbit of $\mathcal{O}_{L}^{K_{u}^{1}}$ with respect to the normal metric is given by

$$
\nabla^{N} V=-[L,[L, \nabla V]]
$$

and hence always admits a double bracket form.

Proof. We let $\pi_{i}$ be the projection onto ker $\left[L_{0},\right]^{\perp} \oplus \omega_{L}$ with respect to the $H^{0}$ metric. Using the invariance of the inner product $\langle.,$.$\rangle , we have for all X \in T \mathcal{O}_{L}^{K_{u}^{1}}$

$$
\begin{aligned}
-d V \cdot X & =-\langle\nabla V, X\rangle \\
& =-\left\langle\nabla V, \operatorname{ad}_{L} \operatorname{ad}_{L}^{-1} X\right\rangle \\
& =\left\langle\operatorname{ad}_{L} \nabla V, \operatorname{ad}_{L}^{-1} X\right\rangle \\
& =\left\langle\pi_{i} \operatorname{ad}_{L} \nabla V, \operatorname{ad}_{L}^{-1} X\right\rangle
\end{aligned}
$$

where in the last step we use the fact that $\operatorname{ad}_{L}^{-1} X \in \operatorname{ker}\left[L_{0}, \cdot\right]^{\perp} \oplus \omega_{L}$. 
Furthermore, the definition of $\operatorname{ad}_{L}^{-1}$ gives

$$
\begin{aligned}
\left\langle\pi_{i} \operatorname{ad}_{L} \nabla V, \operatorname{ad}_{L}^{-1} X\right\rangle & =\left\langle\operatorname{ad}_{L}^{-1} \operatorname{ad}_{L} \pi_{i} \operatorname{ad}_{L} \nabla V, \operatorname{ad}_{L}^{-1} X\right\rangle \\
& =\left\langle\operatorname{ad}_{L} \pi_{i} \operatorname{ad}_{L} \nabla V, X\right\rangle_{N}
\end{aligned}
$$

By the non-degeneracy of $\langle., .\rangle_{N}$ and Equation (13), we conclude that $\nabla^{N} V=$ $\operatorname{ad}_{L} \pi_{i} \operatorname{ad}_{L} \nabla V$. Because $\left[L_{0}, \cdot\right]$ is a skew-adjoint operator then

$$
\operatorname{im}\left[L_{0}, \cdot\right] \subset \operatorname{ker}\left[L_{0}, \cdot\right]^{\perp} .
$$

Together with Equation (6), this shows that for any $\nabla V, \operatorname{ad}_{L} \nabla V \in \operatorname{ker}\left[L_{0}, \cdot\right]^{\perp} \oplus \omega_{L}$ and so $\pi_{i} \operatorname{ad}_{L} \nabla V=\operatorname{ad}_{L} \nabla V$.

3.3. The periodic Toda lattice. Building upon the above result, we describe a canonical way for obtaining the periodic Toda lattices as gradient flows. To this end, recall that $C_{\text {aff }}$ (resp. C) is the Cartan matrix of $\tilde{\mathfrak{g}}$ (resp. $\mathfrak{g}$ ).

The periodic Toda lattice associated with $\mathfrak{g}$ is defined [AvM80] to be the dynamical system $\dot{L}_{p}=\left[L_{p}, B\left(L_{p}\right)\right]$ with

$$
\begin{aligned}
& L_{p}=\sum_{i=1}^{l} b_{i} H_{i}+\sum_{i=1}^{l} a_{i}\left(e_{i}+e_{-i}\right)+a_{0}\left(e_{\gamma} z^{-1}+e_{-\gamma} z\right) \text { and } \\
& \quad B\left(L_{p}\right)=\sum_{i=1}^{l} a_{i}\left(e_{i}-e_{-i}\right)+a_{0}\left(e_{-\gamma} z-e_{\gamma} z^{-1}\right) .
\end{aligned}
$$

The non-periodic Toda lattice is similarly defined as a Lax pair with

$$
L_{n p}=\sum_{i=1}^{l} b_{i} H_{i}+\sum_{i=1}^{l} a_{i}\left(e_{i}+e_{-i}\right) \text { and } B_{n p}\left(L_{n p}\right)=\sum_{i=1}^{l} a_{i}\left(e_{i}-e_{-i}\right) .
$$

\section{Proposition 4.}

Let $x=\left(x_{0}, \ldots, x_{l}\right) \in \mathbb{R}^{l+1} ; x$ is in the kernel of $C_{\text {aff }}$ if and only if $\sum_{i=1}^{l} x_{i} H_{i}-$ $x_{0} H_{\gamma}=0$

Proof. Let $c_{i}$ denote the $i^{\text {th }}$ row of $C_{\text {aff. }}$. The simple roots $\alpha_{i}$ form a basis of $\left(\mathbb{R}^{l}\right)^{*}$ and thus the equations

$$
\begin{aligned}
\alpha_{i}\left(\sum_{j=1}^{l} x_{j} H_{j}-x_{0} H_{\gamma}\right) & =\sum_{j=1}^{l} x_{j} \alpha_{i}\left(H_{j}\right)-x_{0} \alpha_{i}\left(H_{\gamma}\right) \\
& =\sum_{j=0}^{l} c_{i j} x_{j}=c_{i} \cdot x=0
\end{aligned}
$$

are linearly independent for $i=1 \ldots l$. Since $C_{\text {aff }}$ is of corank 1 we have that $\sum_{j=0}^{l} c_{0 j} x_{j}=0$ and $x \in \operatorname{ker} C_{\text {aff }}$.

Proposition 5. There is a unique $x=\left(x_{0}, \ldots, x_{l}\right) \in \mathbb{R}^{l+1}$ which satisfies the equation

$$
\left(C_{\text {aff }}+E_{11}\right) x=-\mathbf{1}
$$


and it has the property that $x_{0}=-c_{\mathfrak{g}}$.

Proof. First, assume that $C_{\text {aff }}+E_{11}$ is not of full rank. Because the lower $l$ rows of $C_{\text {aff }}$ are linearly independent and $C_{\text {aff }}$ is of corank 1 , the vectors $c_{0}$ and $c_{0}+[1,0, \ldots, 0]$ can be represented uniquely as linear combinations of $c_{1}, \ldots, c_{l}$ and hence so can $[1,0, \ldots, 0]$. This in turn implies that $[0, \ldots, 0] \in \mathbb{R}^{l}$ can be written as a non-zero linear combination of the rows of $C$, which is impossible.

For the second part of the statement we have that, by definition of $C_{\text {aff }}$, the first equation of (14) reads

$$
\begin{aligned}
-1 & =\sum_{i=0}^{l} x_{i} \alpha_{0}\left(H_{i}\right)+x_{0} \\
& =3 x_{0}+\sum_{i=1}^{l} x_{i} \alpha_{0}\left(H_{i}\right),
\end{aligned}
$$

where we used the fact that $\alpha_{0}\left(H_{0}\right)=2$. Now recall that $\alpha_{0}=\delta-\gamma$, where $\gamma$ is the highest root for the system of positive roots $\alpha_{1}, \ldots \alpha_{l}$, and if we write $\gamma=\sum_{j=1}^{l} m_{j} \alpha_{j}$ then $c_{\mathfrak{g}}=1+\sum_{j} m_{j}$. Hence

$$
\begin{aligned}
-1 & =3 x_{0}-\sum_{i} x_{i}\left(\sum_{j} m_{j} \alpha_{j}\left(H_{i}\right)\right) \\
& =3 x_{0}-\sum_{j} m_{j} \sum_{i=1}^{l} x_{i} \alpha_{j}\left(H_{i}\right) .
\end{aligned}
$$

The remaining $l$ equations of (14) yield for $j=1 \ldots l$

$$
-1-x_{0} \alpha_{j}\left(H_{0}\right)=\sum_{i=1}^{l} x_{i} \alpha_{j}\left(H_{i}\right)
$$

Plugging this into the first equation, we obtain

$$
\begin{aligned}
-1 & =3 x_{0}-\left(1-c_{\mathfrak{g}}-x_{0} \sum_{j} m_{j} \alpha_{j}\left(H_{0}\right)\right) \\
& =3 x_{0}-\left(1-c_{\mathfrak{g}}+x_{0} \alpha_{0}\left(H_{0}\right)\right) \\
& =3 x_{0}-\left(1-c_{\mathfrak{g}}+2 x_{0}\right),
\end{aligned}
$$

hence $x_{0}=-c_{\mathfrak{g}}$ as announced.

In the following we omit the factor $i=\sqrt{-1}$ for symmetric elements in $\mathfrak{k}_{u}$.

Proof. [Proof of Theorem 1] The function $\langle L, N\rangle$ is a linear function and its gradient with respect to $\langle.,$.$\rangle is therefore N$. Notice that we can ignore the central extension term of $N$. According to Theorem 2, the gradient flow with respect to the normal metric is then

$$
\frac{d}{d t} L=[L,[L, N]]
$$


Because $H_{0}=1 \mathrm{c}-H_{\gamma}$ and $H_{\gamma} \in \operatorname{span}\left\{H_{1}, \ldots H_{l}\right\}$, it is enough to check that

$$
B\left(L_{p}\right)=[L, N] \text {. }
$$

It was shown in [BBR92] that $B_{n p}\left(L_{n p}\right)=\left[L_{n p}, N_{n p}\right]$ with $N_{n p}=\sum_{i=1}^{l} u_{i} H_{i}$ and $u$ satisfying $C u=-\mathbf{1}$. Observe that

$$
\begin{aligned}
L & =L_{n p}+b_{0} H_{0}+a_{0}\left(e_{-\gamma} z+e_{\gamma} z^{-1}\right) \\
B(L) & =B_{n p}\left(L_{n p}\right)+a_{0}\left(e_{-\gamma} z-e_{\gamma} z^{-1}\right)
\end{aligned}
$$

Let $\bar{x} \in \operatorname{ker} C_{\text {aff }}$ be not identically zero. Because $H_{1}, \ldots, H_{l}$ are linearly independent, we conclude using Proposition 4 that $\bar{x}_{0} \neq 0$; so we can assume that $\bar{x}_{0}=1$. Consequently, $y \triangleq x+c_{\mathfrak{g}} \bar{x}$ satisfies $\left(C_{\text {aff }}+E_{11}\right) y=\left[-1+c_{\mathfrak{g}},-1, \ldots,-1\right]^{T}$ and $y_{0}=0$. Hence $C y_{[1 . . l]}=\mathbf{- 1}$ and, using Proposition 4 again and ignoring the central extension term in $H_{0}$, we can write

$$
\begin{aligned}
N & =\sum_{i=0}^{l} x_{i} H_{i}+x_{0} z \frac{d}{d z}+c_{\mathfrak{g}} \sum_{i=0}^{l} \bar{x}_{i} H_{i} \\
& =\sum_{i=1}^{l} y_{i} H_{i}+x_{0} z \frac{d}{d z}=N_{n p}-c_{\mathfrak{g}} z \frac{d}{d z} .
\end{aligned}
$$

By definition of $u$, we see that $\left[e_{\gamma}, \sum_{i} u_{i} H_{i}\right]=-\left(\sum_{j} m_{j} \sum_{i} u_{i} \alpha_{j}\left(H_{i}\right)\right) e_{\gamma}=$ $\sum_{j} m_{j} e_{\gamma}=\left(c_{\mathfrak{g}}-1\right) e_{\gamma}$. Gathering these results, we can evaluate $[L, N]=\left[L, N_{n p}\right]+$ $\left[L,-c_{\mathfrak{g}} z \frac{d}{d z}\right]$ :

$$
\begin{aligned}
{\left[L, N_{n p}\right] } & =B_{n p}\left(L_{n p}\right)+\left[a_{0}\left(e_{\gamma} z^{-1}+e_{-\gamma} z\right), \sum_{i} x_{i} H_{i}\right] \\
& =B_{n p}\left(L_{n p}\right)+a_{0}\left(\left(c_{\mathfrak{g}}-1\right) e_{\gamma} z^{-1}+\left(1-c_{\mathfrak{g}}\right) e_{-\gamma} z\right)
\end{aligned}
$$

and

$$
\left[L,-c_{\mathfrak{g}} z \frac{d}{d z}\right]=-a_{0} c_{\mathfrak{g}}\left(e_{\gamma} z^{-1}-e_{-\gamma} z\right),
$$

which concludes the proof.

4. The periodic Toda flows for simple Lie algebras. In this section, we give an explicit description of the periodic Toda flows. With a mild abuse of notation, we write $e_{i}=E_{i i}-E_{i+1, i+1}$ to indicate that $e_{i}$ is the one-dimensional vector space spanned by $E_{i i}-E_{i+1, i+1}$.

4.1. $\tilde{A}_{l}$. This is the Lie algebra of complex traceless matrices of size $(l+1) \times(l+1)$. We take $\mathfrak{h}$ to be the set of traceless diagonal matrices and we denote by $d_{i}$ the functional that extracts the $i^{\text {th }}$ diagonal element of a matrix. A set of simple roots is given by $\left\{\alpha_{1}=d_{1}-d_{2}, \ldots, \alpha_{l}=d_{l}-d_{l+1}\right\}$ and the corresponding coroots are $\left\{H_{1}=E_{11}-E_{22}, \ldots, H_{l}=E_{l, l}-E_{l+1, l+1}\right\}$. The simple root spaces are given by 
$e_{i}=E_{i, i+1}$. The highest root is $\gamma=\sum_{i=1}^{l} \alpha_{i}=d_{1}-d_{l+1}$ and the corresponding root space is $e_{0}=E_{1, l+1}$. The Coxeter number of $A_{l}$ is $l+1$ and

$$
N=\operatorname{diag}\left(-\frac{l}{2},-\frac{l-2}{2}, \ldots, \frac{l-2}{2}, \frac{l}{2}\right)-(l+1) z \frac{d}{d z} .
$$

ExAmple $4\left(\tilde{A}_{5}\right)$.

The Cartan matrix is

$$
C_{\text {aff }}=\left[\begin{array}{rrrrrr}
2 & -1 & 0 & 0 & 0 & -1 \\
-1 & 2 & -1 & 0 & 0 & 0 \\
0 & -1 & 2 & -1 & 0 & 0 \\
0 & 0 & -1 & 2 & -1 & 0 \\
0 & 0 & 0 & -1 & 2 & -1 \\
-1 & 0 & 0 & 0 & -1 & 2
\end{array}\right] .
$$

We have $N=\operatorname{diag}\left(-\frac{5}{2},-\frac{3}{2},-\frac{1}{2}, \frac{1}{2}, \frac{3}{2}, \frac{5}{2}\right)-6 z \frac{d}{d z}$ and

$$
\begin{gathered}
L=\left[\begin{array}{cccccc}
b_{1}-b_{0} & a 1 & 0 & 0 & 0 & \frac{a_{0}}{z} \\
a_{1} & b_{2}-b_{1} & a_{2} & 0 & 0 & 0 \\
0 & a_{2} & b_{3}-b_{2} & a_{3} & 0 & 0 \\
0 & 0 & a_{3} & b_{4}-b_{3} & a_{4} & 0 \\
0 & 0 & 0 & a_{4} & b_{5}-b_{4} & a_{5} \\
a_{0} z & 0 & 0 & 0 & a_{5} & b_{0}-b_{5}
\end{array}\right] \oplus b_{0} \mathrm{c}, \\
0 \\
0
\end{gathered}
$$

We obtain $\dot{b}_{i}=-2 a_{i}^{2}$ and $\dot{V}=2 \sum a_{i}^{2}$.

4.2. $\tilde{B}_{l}$. This is the orthogonal Lie algebra in odd dimensions, $\mathfrak{o}(2 l+1)$. We take $\mathfrak{h}$ to be the space of matrices of the form $\operatorname{diag}\left(0, f_{1},-f_{1}, f_{2},-f_{2}, \ldots, f_{l},-f_{l}\right)$ and we denote by $d_{i}$ the functional that extracts the $2 i^{t h}$ diagonal element of $h \in \mathfrak{h}$. A set of simple roots is given by $\left\{d_{1}-d_{2}, \ldots, d_{l-1}-d_{l}, d_{l}\right\}$ and the corresponding coroots are $\left\{H_{1}=E_{22}-E_{33}-E_{44}+E_{55}, H_{2}=E_{44}-E_{55}-E_{66}+E_{77} \ldots H_{l-1}=\right.$ $\left.E_{2(l-1), 2(l-1)}-E_{2 l-1,2 l-1}-E_{2 l, 2 l}+E_{2 l+1,2 l+1}, H_{l}=2 E_{2 l, 2 l}-2 E_{2 l+1,2 l+1}\right\}$. The simple root spaces are $\left\{e_{i}=E_{2 i, 2(i+1)}-E_{2(i+1)+1,2 i+1}\right.$ for $\left.i \neq l ; e_{l}=\sqrt{2}\left(E_{2 l, 1}-E_{1,2 l+1}\right)\right\}$ The highest root is $\gamma=\alpha_{1}+2\left(\sum_{i=2}^{l} \alpha_{l}\right)=d_{1}+d_{2}$ and the corresponding root space is $e_{0}=E_{4,3}-E_{2,5}$. The Coxeter number of $B_{l}$ is $2 l$ and

$$
N=\operatorname{diag}(0,-l, l,-(l-1), l-1, \ldots,-1,1)-2 l z \frac{d}{d z} .
$$


4.3. $\tilde{C}_{l}$. This is the symplectic algebra $\mathfrak{s p}(l)$, i.e. the space of $2 l \times 2 l$ matrices $M$ satisfying $M^{T} J+J M=0$ where $J=\left[\begin{array}{cc}0 & I \\ -I & 0\end{array}\right]$.

We take $\mathfrak{h}$ to be the space of matrices of the form $\operatorname{diag}\left(f_{1},-f_{1}, \ldots, f_{l},-f_{l}\right)$ and we denote by $d_{i}$ the functional that extracts the $(2 i-1)^{t h}$ diagonal element of $h \in \mathfrak{h}$. A set of simple roots is given by $\left\{\alpha_{1}=d_{1}-d_{2}, \ldots, \alpha_{l-1}=d_{l-1}-d_{l}, \alpha_{l}=2 d_{l}\right\}$ and the corresponding coroots are $\left\{H_{1}=E_{11}-E_{22}-E_{33}+E_{44}, H_{2}=E_{33}-E_{44}-E_{55}+\right.$ $E_{66} \ldots H_{l-1}=E_{2(l-1)-1,2(l-1)-1}-E_{2 l-2,2 l-2}-E_{2 l-1,2 l-1}+E_{2 l, 2 l}, H_{l}=E_{2 l-1,2 l-1}-$ $\left.E_{2 l, 2 l}\right\}$. The simple root spaces are $\left\{e_{i}=E_{2 i-1,2(i+1)-1}-E_{2(i+1), 2 i}\right.$ for $i \neq l ; e_{l}=$ $\left.E_{2 l-1,2 l}\right\}$. The highest root is $\gamma=2\left(\sum_{i=1}^{l-1} \alpha_{l}\right)+\alpha_{l}=2 d_{1}$ and the corresponding root space is $e_{0}=E_{1,2}$. The Coxeter number of $B_{l}$ is $2 l$ and

$$
N=\operatorname{diag}\left(-\frac{(2 l-1)}{2}, \frac{(2 l-1)}{2},-\frac{(2 l-3)}{2}, \frac{(2 l-3)}{2}, \ldots, \frac{3}{2},-\frac{1}{2}, \frac{1}{2}\right)-2 l z \frac{d}{d z} .
$$

4.4. $\tilde{D}_{l}$. This is the orthogonal Lie algebra in even dimensions, $\mathfrak{o}(2 l)$. As for $C_{l}$, we take $\mathfrak{h}$ to be the space of matrices of the form $\operatorname{diag}\left(f_{1},-f_{1}, \ldots, f_{l},-f_{l}\right)$ and we denote by $d_{i}$ the functional that extracts the $(2 i-1)^{t h}$ diagonal element of $h \in \mathfrak{h}$. A set of simple roots is given by $\left\{\alpha_{1}=d_{1}-d_{2}, \ldots, \alpha_{l-1}=d_{l-1}-d_{l}, \alpha_{l}=d_{l-1}+\right.$ $\left.d_{l}\right\}$ and the corresponding coroots are $\left\{H_{1}=E_{11}-E_{22}-E_{33}+E_{44}, H_{2}=E_{33}-\right.$ $E_{44}-E_{55}+E_{66} \ldots H_{l-1}=E_{2(l-1)-1,2(l-1)-1}-E_{2 l-2,2 l-2}-E_{2 l-1,2 l-1}+E_{2 l, 2 l}, H_{l}=$ $\left.E_{2(l-1)-1,2(l-1)-1}-E_{2 l-2,2 l-2}+E_{2 l-1,2 l-1}-E_{2 l, 2 l}\right\}$. The simple root spaces are $\left\{e_{i}=E_{2 i-1,2(i+1)-1}-E_{2(i+1), 2 i}\right.$ for $\left.i \neq l ; e_{l}=E_{2 l-3,2 l}-E_{2 l-1,2 l-2}\right\}$ The highest root is $\gamma=d_{1}-d_{2}+2\left(\sum_{i=2}^{l-2} \alpha_{l}\right)+\alpha_{l-1}+\alpha_{l}=d_{1}+d_{2}$ and the corresponding root space is $e_{0}=E_{1,4}-E_{3,2}$. The Coxeter number of $B_{l}$ is $2 l-2$ and

$$
N=\operatorname{diag}(-(l-1), l-1,-(l-2), l-2, \ldots,-1,1,0,0)-(2 l-2) z \frac{d}{d z} .
$$

5. Acknowledgments. I would like to thank Roger Brockett for introducing me to the world of completely integrable systems and the anonymous reviewers for their very helpful suggestions on an earlier version of this manuscript.

\section{REFERENCES}

[Ad179] M. AdLer, On a trace functional for pseudo-differential operators and the symplectic structure of Korteveg-Devries type equations., Inventiones Mathematicae, 50 (1979), pp. 219-248.

[AP83] M.F. Atiyah And A.N Pressley, Convexity and loop groups, Arithmetic and Geometry, vol. II, vol. 36, Birkhäuser, Boston, 1983, pp. 33-64.

[Ati82] M.F. Атіуан, Convexity and commuting hamiltonians, Bull. London Math. Soc., 16 (1982), pp. 1-15.

[AvM80] M. Adler and P. VAn Moerbeke, Completely integrable systems, euclidean lie algebras and curves, Advances in Math., 38 (1980), pp. 267-317. 
[BB06] M.A. Belabbas AND R.W. Brockett, Input-output hamiltonian systems and neural signal processing, Proceedings of the 46th IEEE Conference on Decision and Control, 2006, pp. 3351-3356.

[BBR90] A.M. Bloch, R. Brockett, and T. Ratiu, A new formulation of the generalized Toda lattice equations and their fixed point analysis via the moment map, Bulletin of the AMS, (1990), no. 2, pp. 447-456.

[BBR92] A.M. Bloch, R.W. Brockett, and T. Ratiu, Completely integrable gradient flows, Commun. Math. Phys., 147 (1992), pp. 57-74.

[BC97] A.M. Bloch AND P.E. Crouch, Optimal control and the full Toda flow, Proceedings of the 36th IEEE Conference on Decision and Control, vol. 2, Dec. 1997, pp. 1736-1740.

[Bog76] O.I. Bogoyavlensky, On perturbation of the periodic Toda lattice, Comm. Math. Phys., 5 (1976), pp. 201-209.

[Bou72] N. Bourbaki, Groupes et algèbres de Lie, chap. 2 83 3, Hermann, 1972.

[Bro76] R.W. Brockett, Some geometric questions in the theory of linear systems, IEEE Trans. Automatic Control AC-21, (1976), no. 4, pp. 449-455.

[Bro89] Least squares matching problems, Linear Algebra and its Applications, 122/123/124 (1989), pp. 761-777.

[Bro91] D D D _ D _ namical systems that sort lists and solve linear programming problems, Linear Algebra and its applications, 146 (1991), pp. 79-91.

[Bro93] D Differential geometry and the design of gradient algorithms, Proceedings of Symposia in Pure Mathematics (R. Green and S. T. Yau, eds.), vol. 54, 1993, pp. 69-92.

[Bro94] The double bracket equation as a solution to a variational problem, Fields Inst. Comm., 3 (1994), pp. 69-76.

[Bro97] _ A rational flow for the periodic Toda lattice equations, Operators, Systems and Linear Algebra (U. Helmke et al., ed.), B. G. Teubner, Stuttgart, 1997.

[Bro99] Explicitly solvable control problems with nonholonomic constraints, Proc. 38th IEEE Conf. on Dec. and Control (Phoenix, AZ), 1999, pp. 13-16.

[BT95] M. Blau and G. Thompson, On diagonalization in Map $(m, g)$, Comm. Math. Phys., 171 (1995), pp. 639-660.

[Dei99] P.A. DeIFT, Orthogonal polynomials and random matrices, AMS, Providence RI, 1999.

[Fay88] L.E. Faybusovich, Explicitly solvable optimal control problems, Int. J. Control., 48:6 (1988), pp. 235-250.

[Fla74] H. Flaschka, The Toda lattice I 8 II, Phys. Rev. B, 9 (1974), pp. 1924-1925.

[HM96] U. Helmke and J.B. Moore, Optimization and dynamical systems, Springer, 1996.

[Kac94] V.G. KAC, Infinite dimensional Lie algebras, Cambridge University Press, 1994.

[Kos79] B. Kostant, The solution to a generalized Toda lattice and representation theory, Adv. Math., 39 (1979), pp. 195-338.

[Kri79] P.S. Krishnaprasad, Symplectic mechanics and rational functions, Ricerche Automat., 10 (1979), pp. 107-135.

[Mos] J. Moser, Finitely many mass points on the line under the influence of an exponential potential, Batelles Rencontres, Lecture Notes in Physics, Springer, pp. 417-497.

[PS88] A. Pressley and G. Segal, Loop groups, Oxford University Press, 1988.

[RS94] A Reyman and M. Semenov-Tian-Shansky, Group-theoretic methods in the theory of finite-dimensional integrable systems, Dynamical systems VIIm (V.I. Arnold and S.P. Novikov, eds.), Encyclopaedia of Mathematical Sciences, vol. 16, Springer-Verlag, 1994, pp. 116-225.

[Sam90] H. SAmelson, Notes on lie algebras, Springer, 1990.

[Seg79] G.B. Segal, The topology of spaces of rational functions, Acta Math., 143 (1979), pp. 39-72.

[Seg81] Unitary representations of some infinite-dimensional groups, Comm. Math. 
Phys., 80 (1981), pp. 301-342.

[Sym80] W.V. Symes, Hamiltonian group actions and integrable systems, Phys. D, 1 (1980), pp. 339-376. 